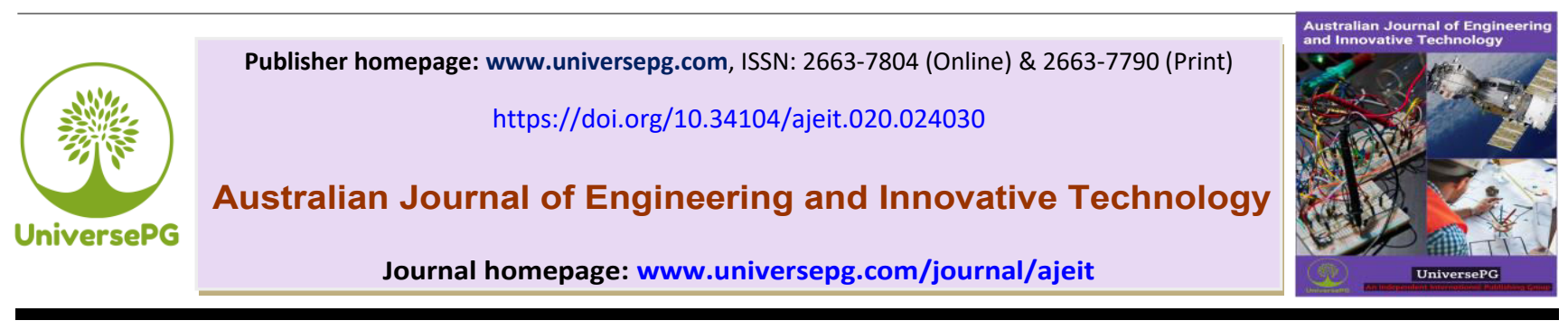

\title{
One Dimensional Heat Transfer through a Uniform Plane Wall by Using Finite Volume Method
}

\author{
Saiful Islam ${ }^{1}$, Md. Sirajul Islam² ${ }^{2}$ and Sajib Mandal ${ }^{3}$ \\ ${ }^{1}$ Department of Computer Science and Engineering, Stamford University Bangladesh, Dhaka, Bangladesh; and \\ ${ }^{2 \& 3}$ Department of Mathematics, Bangabandhu Sheikh Mujibur Rahman Science and Technology University, Gopalganj, \\ Bangladesh. \\ *Correspondence: saiful.islam@stamforduniversity.edu.bd
}

\begin{abstract}
In this paper, finite volume method has been used to investigate one dimensional conductive heat transfer throw a uniform plane wall. Then the step by step procedures of this numerical solution are described and implemented in a real world problem where tri-diagonal matrix algorithm and Gaussian elimination matrix method are applied to solve the system of our discretized algebraic system of equations. Finally, to check the accuracy of our method, a comparison between the numerical solution obtained by finite volume techniques and exact solution is presented which show a minimum error compared to other existing methods.
\end{abstract}

Keywords: Computational fluid dynamics, Finite volume method, and Tri-diagonal matrix algorithm.

\section{INTRODUCTION:}

Nowadays, to investigate the mass, momentum, and heat transfer phenomena, computational fluid dynamics (CFD) study numerical solutions which is associated with the phenomena of thermodynamics, turbo machinery, chemical manufacturing, power generation, weather simulation, biological engineering, meteorology, aerospace, reaction chemistry, predicting fluid flow and heat transfer (Islam et al., 2019). Though the fundamental source of almost all CFD problems is Navier-Stokes equations which describe many single-phase (liquid or gas, but not both) fluid flows.

Recently, due to the revolution of computer technology, abundant computational grid techniques have been developed which is very efficient to solve numerous engineering problems (Cheniguel and Reghioua, 2013; Chuathong and Toutip, 2011). Among these numerical grid techniques, finite difference method (FDM) is used as a common numerical technique to solve numerous engineering problems (Lau and Kuruganty, 2010). Again, finite element method (FEM) is another kind of commonly used numerical technique which has been applied to solve many heat transfer problems (Patil and Prasad, 2013). A generalized transfer equation for a dependent variable $\phi$ which can be mass, concentration, heat and momentum is given by Patankar (1980), where discretization technique is applied for CFD analysis (Islam and Hossain, 2019).

Moreover, the finite volume method (FVM) is becoming most popular numerical technique which is commonly used in commercial CFD software such as COMSOL Multiphysics (Versteeg and Malalasekera, 2007). Though to solve any problem these three methods have its own merits besides its demerits, FVM technique is one of the utmost flexible and multipurpose techniques to solve CFD (Uddin et al., 2020). In this paper, we have described an engineering problem by the point of view of FVM. Due to its wide-spread popularity in CFD, this method has been applied previously in different mechanical engineering problem as like thermo elastic and linear elastic problems (Berezovski and 
Maugin, 2001; Demirdžić and Muzaferija, 1994; and Jasak and Weller, 2000).

The rest of our paper is prepared as follows. A short review of FVM with the help of Tri-Diagonal Matrix Algorithm TDMA is given in Section 2. In Section 3 , the numerical solutions obtained by this technique is given where a comparison between exact and our numerical solutions is also described. Finally, in Section 5 we have concluded this paper.

\section{FINITE VOLUME METHOD:}

Consider the steady state diffusion of a property $T$ in a one-dimensional domain defined in Fig 1. The process is governed by

$$
\frac{d}{d x}\left(\psi \frac{d T}{d x}\right)+q=0
$$

Where $\psi$ is the thermal connectivity, $T$ is the temperature and $q$ is the source term (i.e., the rate of heat generation per unit volume).

\subsection{Grid Generation}

In the finite volume method first of all, we have to divide the domain into discrete control volumes. Let us consider a number of nodal points in the domain space from $A$ to $B$. The boundaries (or faces) of control volumes are positioned mid-way between adjacent nodes. Thus each node is surrounded by a control volume or cell. Today it is common practice to set up control volumes near to the edge of the domain in such a way where the physical boundaries coincide with the control volume boundaries. At this point it is appropriate to establish a system of notation that can be used in future developments.

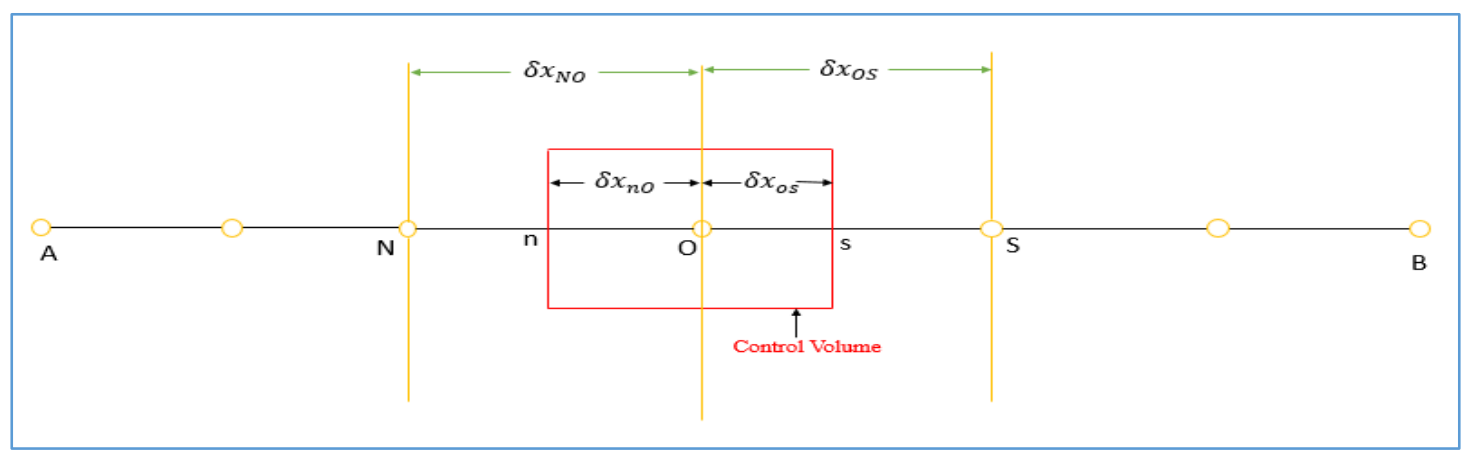

Fig 1: Grid point generation for finite volume method with control volume.

A general nodal point is identified by $O$ and its neighbor's nodes to the north and south are represented by $N$ and $S$ respectively. The south side control volume face is mentioned by $s$ and the north side face of the control volume is mentioned to by $n$. The distances between the nodes $O$ and $S$, and between nodes $N$ and $O$ are represented by $\delta x_{O S}$ and $\delta x_{N O}$ respectively. In the same way, distances between point $O$ and face $s$ is denoted by $\delta x_{O S}$ and distances between face $n$ and point $O$ is denoted by $\delta x_{n o}$.

\subsection{Discretization}

To discretize the governing equation, we will integrate the governing equation (1) over the control volume at its nodal point $P$.

$$
\int_{\Delta V} \frac{d}{d x}\left(\psi \frac{d T}{d x}\right) d V+\int_{\Delta V} q d V=0
$$

Then we get, $\left(\psi A \frac{d T}{d x}\right)_{S}-\left(\psi A \frac{d T}{d x}\right)_{n}+q A \delta x=0$

Where $A$ the cross-sectional area of the control volume face is, $\Delta V$ is the volume and $q$ is the source term over the control volume. This is a very attractive feature of this finite volume method where the discretized equation contains a clear physical interpretation. Equation 2 states that the diffusive flux of $T$ leaving the south face minus the diffusive flux of $T$ entering the north face is equal to the generation of $T$ over the control volume. In order to derive useful forms of the discretized equations, the interface diffusion coefficient $\psi$ and the gradient $\frac{d T}{d x}$ at west $n$ and east $s$ are required. And the diffusive flux terms are evaluated as -

$$
\begin{aligned}
& \left(\psi A \frac{d T}{d x}\right)_{S}=\psi_{s} A\left(\frac{T_{S}-T_{O}}{\delta x_{O S}}\right) \\
& \left(\psi A \frac{d T}{d x}\right)_{n}=\psi_{n} A\left(\frac{T_{O}-T_{N}}{\delta x_{N O}}\right)
\end{aligned}
$$


For practical situations, the source term $q$ may be a function of the dependent variable. By substituting the values of equations (3) and (4) into equation (2), we get

$\psi_{S} A\left(\frac{T_{S}-T_{O}}{\delta x_{O S}}\right)-\psi_{n} A\left(\frac{T_{O}-T_{N}}{\delta x_{N O}}\right)+q A \delta x=0$

By rearranging equation (5), we can write

$$
\begin{aligned}
& \left(\frac{\psi_{S} A}{\delta x_{O S}}+\frac{\psi_{n} A}{\delta x_{N O}}\right) T_{O}=\left(\frac{\psi_{n} A}{\delta x_{N O}}\right) T_{N}+\left(\frac{\psi_{S} A}{\delta x_{O S}}\right) T_{S} \\
& +q A \delta x
\end{aligned}
$$

By representing the coefficient of $T_{O}, T_{N}$ and $T_{S}$ as $a_{O}, a_{N}$ and $a_{S}$ respectively then the equation (6) can be written as

$a_{O} T_{O}=a_{N} T_{N}+a_{S} T_{S}+S_{u}$

Where (here $\delta x_{N O}=\delta x_{O S}=\delta x$ )

\begin{tabular}{|c|c|c|c|c|}
\hline $\boldsymbol{a}_{\boldsymbol{N}}$ & $\boldsymbol{a}_{\boldsymbol{S}}$ & $\boldsymbol{a}_{\boldsymbol{o}}$ & $\boldsymbol{S}_{\boldsymbol{o}}$ & $\boldsymbol{S}_{\boldsymbol{u}}$ \\
\hline$\frac{\boldsymbol{\psi}_{\boldsymbol{n}} \boldsymbol{A}}{\boldsymbol{\delta} \boldsymbol{x}}$ & $\frac{\psi_{S} A}{\delta x}$ & $a_{N}+a_{S}-S_{O}$ & 0 & $q A \delta x$ \\
\hline
\end{tabular}

But there will be a little difference between the boundary conditions at first node (at point $A$ ) and for last node (at point $B$ ). In this case, linear approximation will be use between the adjacent nodal point and the boundary. For this reasons, we will introduce the linear approximation for temperatures between $A$ and $O$ as like:

$$
\begin{aligned}
& \psi_{S} A\left(\frac{T_{S}-T_{O}}{\delta x}\right)-\psi_{A} A\left(\frac{T_{O}-T_{A}}{\delta x / 2}\right)+q A \delta x=0 \\
& \Rightarrow\left(\frac{\psi_{S} A}{\delta x}+\frac{\psi_{A} A}{\frac{\delta x}{2}}\right) T_{O}=\left(\frac{\psi_{S} A}{\delta x}\right) T_{S}+\left(\frac{\psi_{A} A}{\frac{\delta x}{2}}\right) T_{A} \\
& +q A \delta x
\end{aligned}
$$

This can be rewrite as,

$a_{O} T_{O}=a_{N} T_{N}+a_{S} T_{S}+S_{u}$

Where

\begin{tabular}{|c|c|c|c|c|}
\hline $\boldsymbol{a}_{\boldsymbol{N}}$ & $\boldsymbol{a}_{\boldsymbol{S}}$ & \multicolumn{1}{c|}{$\boldsymbol{a}_{\boldsymbol{o}}$} & $\boldsymbol{S}_{\boldsymbol{O}}$ & $\boldsymbol{S}_{\boldsymbol{u}}$ \\
\hline $\mathbf{0}$ & $\frac{\psi_{S} A}{\delta x}$ & $\begin{array}{l}a_{N}+a_{S} \\
-S_{O}\end{array}$ & $-\frac{\psi_{A} A}{\delta x / 2}$ & $\begin{array}{l}\left(\frac{\psi_{A} A}{\delta x / 2}\right) T_{A} \\
+q A \delta x\end{array}$ \\
& & & & \\
\hline
\end{tabular}

Similarly, we will introduce the linear approximation for temperatures between $O$ and $B$ as like:

UniversePG I www.universepg.com

$$
\begin{gathered}
\psi_{B} A\left(\frac{T_{B}-T_{O}}{\frac{\delta x}{2}}\right)-\psi_{n} A\left(\frac{T_{O}-T_{N}}{\delta x}\right)+q A \delta x=0 \\
\Rightarrow\left(\frac{\psi_{B} A}{\frac{\delta x}{2}}+\frac{\psi_{n} A}{\delta x}\right) T_{O}= \\
\left(\frac{\psi_{n} A}{\delta x}\right) T_{N}+\left(\frac{\psi_{B} A}{\frac{\delta x}{2}}\right) T_{B}+q A \delta x
\end{gathered}
$$

This can be rewrite as,

$a_{O} T_{O}=a_{N} T_{N}+a_{S} T_{S}+S_{u}$

Where

\begin{tabular}{|c|c|c|c|c|}
\hline $\boldsymbol{a}_{\boldsymbol{N}}$ & $\boldsymbol{a}_{\boldsymbol{S}}$ & \multicolumn{1}{c|}{$\boldsymbol{a}_{\boldsymbol{o}}$} & $\boldsymbol{S}_{\boldsymbol{o}}$ & $\boldsymbol{S}_{\boldsymbol{u}}$ \\
\hline$\frac{\boldsymbol{\psi}_{\boldsymbol{n}} \boldsymbol{A}}{\boldsymbol{\delta} \boldsymbol{x}}$ & 0 & $\begin{array}{l}a_{N}+a_{S} \\
-S_{O}\end{array}$ & $-\frac{\psi_{B} A}{\delta x / 2}$ & $\begin{array}{l}\left(\frac{\psi_{B} A}{\delta x / 2}\right) T_{B} \\
+q A \delta x\end{array}$ \\
\hline
\end{tabular}

When we will get the all discretized equations from equation (7), (9) and (11), to solve a problem we have to set up at each of the nodal points. The resulting system of linear algebraic equations will be solved to obtain the distribution of the property $T$ at each nodal point. There are different types of matrix solution technique (Gaussian Elimination Method, Tri-Diagonal Matrix Algorithm, Gauss-Jordan Elimination, etc.) exist which can be used to solve the system of linear algebraic equations. From this system, we will use Gaussian Elimination (GE) Method (Sasaki and Murao, 1982) and Tri-Diagonal Matrix Algorithm (TDMA) (El-Mikkawy, 2004) to find the values of temperature distributions $T$.

\section{RESULT AND DISCUSSION:}

Consider a plane wall whose thickness is $1 \mathrm{~m}(\mathbf{F i g}$ 2) and this has a source effect in this heat conduction system. The two ends of this wall maintained at constant temperature as like $300^{\circ} \mathrm{C}$ and $800^{\circ} \mathrm{C}$ respectively. The thermal conductivity $\psi$ of this wall is equals to $0.5 \mathrm{~W} / \mathrm{m} . \mathrm{K}$, heat generation $q=1000 \mathrm{~W} / \mathrm{m}^{3}$, and the cross-sectional area $A$ is $10 \mathrm{~m}^{2}$. The main goal of this work is to calculate the temperature distributions of this wall by using our control volume method where we will consider the dimensions of the $Y$-and $Z$-directions are so large. For this reason the temperature gradients has significant change along the $X$-direction only. 


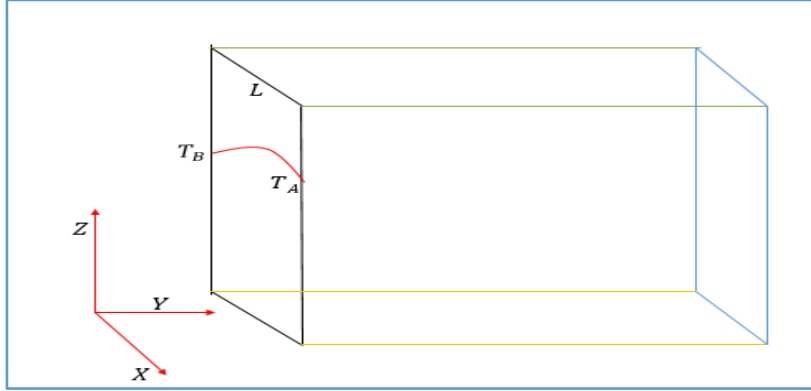

Fig 2: A uniform plane wall of $1 m$ length.

\subsection{Numerical Solution by FVM}

First of all, we have to divide the whole length of the wall into some control volume (along only $X$ directions). In this work, we will calculate the temperature distribution in five internal points. That is, the difference between two control volumes is $0.2 \mathrm{~m}$ (since total length of the wall is $1 \mathrm{~m}$, so $\delta x=1 / 5 m=0.2 m)$.

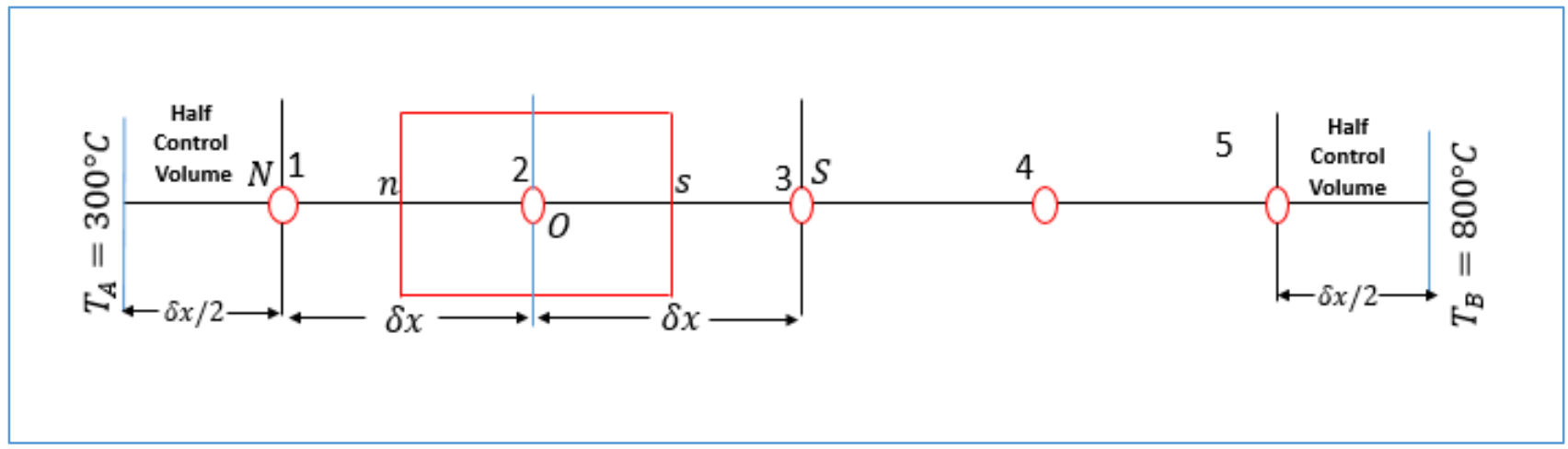

Fig 3: Grid point generation of total domain with control volume and half control volume.

For our given problem (one-dimensional heat conduction) governing equation is,

$\frac{d}{d x}\left(\psi \frac{d T}{d x}\right)+q=0$

Where, $\psi$ is the thermal conductivity, $T$ is the temperature and $q$ is the source term. To get the discretization equation, we have to integrate equation (12) over the control volume. After simplifying (which has already described in Section 2 ) we get as like equation (6),

$$
\begin{aligned}
\left(\frac{\psi_{S} A}{\delta x_{O S}}+\frac{\psi_{n} A}{\delta x_{N O}}\right) T_{O} & =\left(\frac{\psi_{n} A}{\delta x_{N O}}\right) T_{N}+\left(\frac{\psi_{S} A}{\delta x_{O S}}\right) T_{S} \\
+ & q A \delta x
\end{aligned}
$$

By representing the coefficient of $T_{O}, T_{N}$ and $T_{S}$ as $a_{O}, a_{N}$ and $a_{S}$ respectively then the equation (13) can be written as

$a_{O} T_{O}=a_{N} T_{N}+a_{S} T_{S}+S_{u}$

Where (here $\delta x_{N O}=\delta x_{O S}=\delta x$ )

\begin{tabular}{|c|c|c|c|c|}
\hline $\boldsymbol{a}_{\boldsymbol{N}}$ & $\boldsymbol{a}_{\boldsymbol{S}}$ & $\boldsymbol{a}_{\boldsymbol{o}}$ & $\boldsymbol{S}_{\boldsymbol{o}}$ & $\boldsymbol{S}_{\boldsymbol{u}}$ \\
\hline$\frac{\boldsymbol{\psi}_{\boldsymbol{n}} \boldsymbol{A}}{\boldsymbol{\delta} \boldsymbol{x}}$ & $\frac{\psi_{S} A}{\delta x}$ & $a_{N}+a_{S}-S_{O}$ & 0 & $q A \delta x$ \\
\hline
\end{tabular}

In this problem, the given data are

\begin{tabular}{|c|c|}
\hline$L=1 m$ & $\psi_{n}=\psi_{s}=\psi$ \\
$\delta x_{N o}=\delta x_{O S}=\delta x$ & $=0.5 \mathrm{~W} / \boldsymbol{m} . K$ \\
$=\frac{1}{5} \boldsymbol{m}=\mathbf{0 . 2} \boldsymbol{m}$ & $q=1000 \mathrm{~W} / \mathrm{m}^{\mathbf{3}}$ \\
\hline$A_{n}=A_{s}=A$ & $T_{A}=300^{\circ} \mathrm{C}$ \\
$=\mathbf{1 0 ~ \mathrm { m } ^ { 2 }}$ & $T_{B}=800^{\circ} \mathrm{C}$ \\
\hline
\end{tabular}

By using these values, we get $a_{N}=a_{S}=25, a_{O}=$ 50, and $S_{u}=2000$. Again by using the value of $a_{N}$, $a_{S}, a_{O}$ and $S_{u}$ into equation (14), we get the discretized equation for the nodal points 2,3 , and 4 as,

$$
\left.\begin{array}{l}
50 T_{2}=25 T_{1}+25 T_{3}+2000 \\
50 T_{3}=25 T_{2}+25 T_{4}+2000 \\
50 T_{4}=25 T_{3}+25 T_{5}+2000
\end{array}\right\}
$$

On the other hand, at nodal point 1 (at point $A$ ) which has already described in Section 2 the coefficients will be,

\begin{tabular}{|c|c|c|c|c|}
\hline $\boldsymbol{a}_{\boldsymbol{N}}$ & $\boldsymbol{a}_{\boldsymbol{S}}$ & $\boldsymbol{a}_{\boldsymbol{o}}$ & $\boldsymbol{S}_{\boldsymbol{O}}$ & $\boldsymbol{S}_{\boldsymbol{u}}$ \\
\hline $\mathbf{0}$ & $\frac{\psi_{S} A}{\delta x}$ & $\begin{array}{l}a_{N}+a_{S} \\
-S_{O}\end{array}$ & $-\frac{\psi_{A} A}{\delta x / 2}$ & $\begin{array}{l}\left(\frac{\psi_{A} A}{\delta x / 2}\right) T_{A} \\
+q A \delta x\end{array}$ \\
\hline
\end{tabular}

By using the given values, we get $a_{N}=0, a_{S}=$ $25, S_{O}=50, a_{O}=75$, and $S_{u}=17000$. Then the equation (14) can be written as, 
$75 T_{1}=25 T_{2}+17000$

Which is the discretized equation for the nodal point 1. Similarly, at nodal point 5 which are a half control volume and the coefficients will be (which has already described in Section 2),

\begin{tabular}{|c|c|c|c|c|}
\hline $\boldsymbol{a}_{\boldsymbol{N}}$ & $\boldsymbol{a}_{\boldsymbol{S}}$ & $\boldsymbol{a}_{\boldsymbol{o}}$ & $\boldsymbol{S}_{\boldsymbol{o}}$ & $\boldsymbol{S}_{\boldsymbol{u}}$ \\
\hline$\frac{\boldsymbol{\psi}_{\boldsymbol{n}} \boldsymbol{A}}{\boldsymbol{\delta} \boldsymbol{x}}$ & 0 & $a_{N}+a_{S}$ & $-\frac{\psi_{B} A}{\delta x / 2}$ & $\begin{array}{l}\left(\frac{\psi_{B} A}{\delta x / 2}\right) T_{B} \\
+q A \delta x\end{array}$ \\
\hline
\end{tabular}

By using the given values, we get of $a_{N}=25$, $a_{S}=0, S_{O}=50, a_{O}=75$, and $S_{u}=42000$. Then the equation (14) can be written as,

$75 T_{5}=25 T_{4}+42000$

Which is the discretized equation for the nodal point 5 .

Therefore, the set of discretized equations are:

$$
\left.\begin{array}{c}
75 T_{1}=25 T_{2}+17000 \\
50 T_{2}=25 T_{1}+25 T_{3}+2000 \\
50 T_{3}=25 T_{2}+25 T_{4}+2000 \\
50 T_{4}=25 T_{3}+25 T_{5}+2000 \\
75 T_{5}=25 T_{4}+42000
\end{array}\right\}
$$

This system of algebraic linear equation can be solved by different method such as TDMA or Gaussian elimination (GE) method on by hand. But in this we have solved this system of linear equations easily by MATLAB. Then we get our temperature distribution as,

$$
\left[\begin{array}{l}
T_{1} \\
T_{2} \\
T_{3} \\
T_{4} \\
T_{5}
\end{array}\right]=\left[\begin{array}{l}
450 \\
670 \\
810 \\
860 \\
850
\end{array}\right]
$$

\subsection{Exact Solution}

Our governing equation is $\frac{d}{d x}\left(\psi \frac{d T}{d x}\right)+q=0$ where boundary conditions, $T_{A}=300^{\circ} \mathrm{C}$ at $x=0 \mathrm{~m}$, and $T_{B}=800^{\circ} \mathrm{C}, x=1 \mathrm{~m}$. By simplifying this we get our general solution,

$T=C x-\left(\frac{q}{2 \psi}\right) x^{2}+D$

Where $C$ and $D$ are integral constant. By applying the boundary condition we get $C=1500$ and $D=$ 300 , so the complete solution is,

$T=1500 x-\left(\frac{q}{2 \psi}\right) x^{2}+300$
For different values of $x$, we will get the temperature distribution at different nodal points (Table 1).

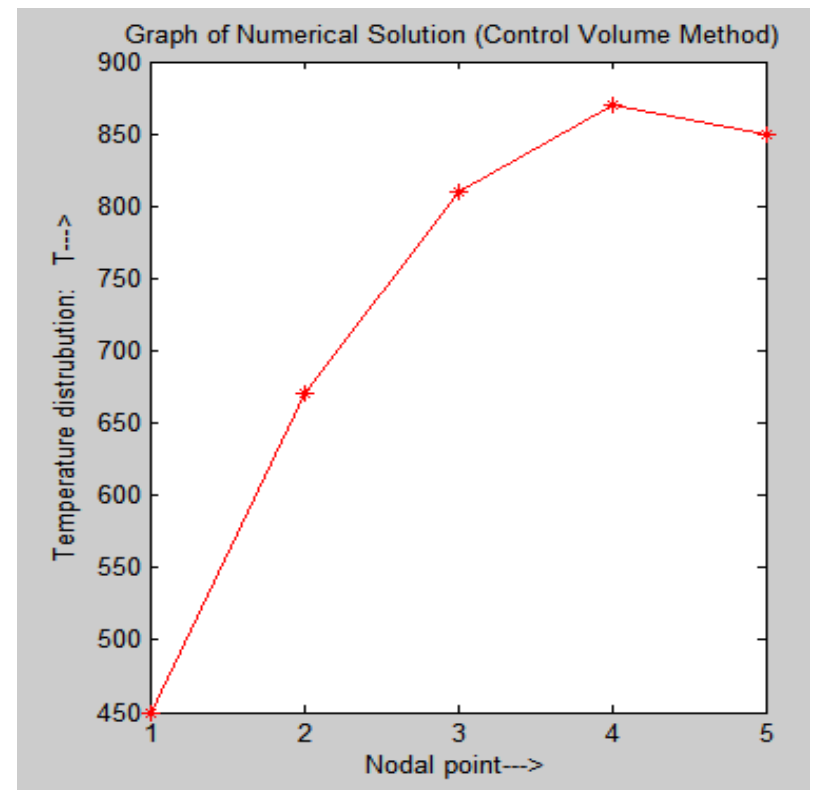

(a)

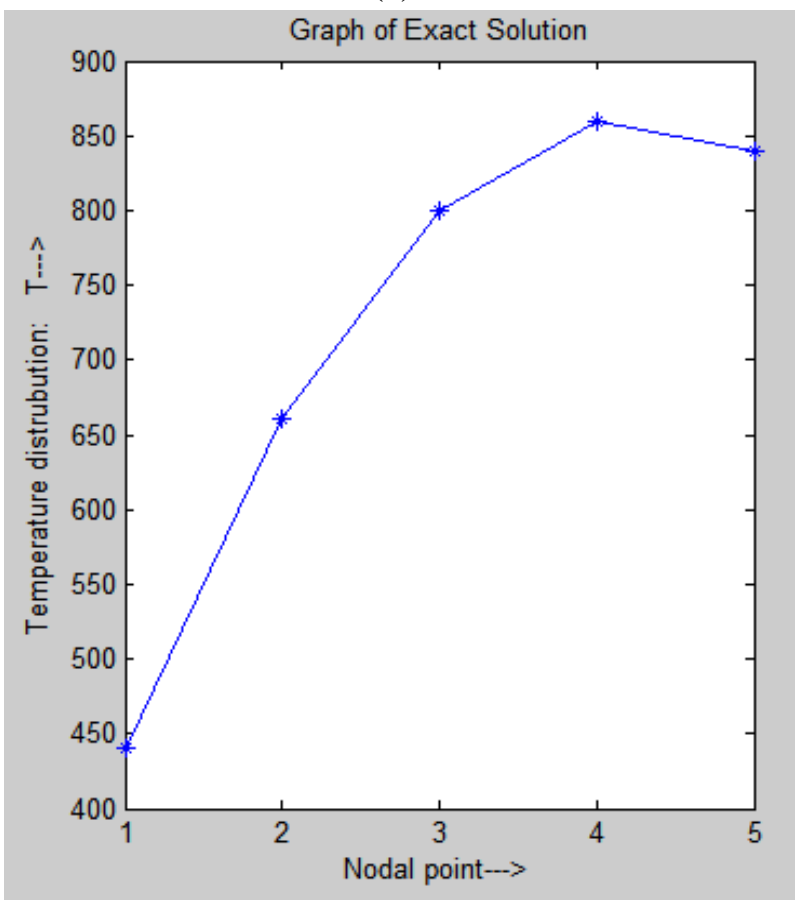

(b)

Fig 4: Graphical representation of (a) Numerical Solution, and (b) Exact Solution.

On the other hand, Fig 4 shows graphical representations of our numerical solution by FVM and exact/analytical solution. Again, Table 2 shows the details presentation of our work where FVM has completed by TDMA and Gaussian elimination (GE) method. Though the two methods show the same result but these numerical solutions have some errors $\left(\frac{\text { Exact Soltution-Numerical Solution }}{\text { Exact Solution }}\right)$. 
But if we divide the domain into more and more control volume, the error will be minimized than the before.

Table 1: Exact/Analytic solutions for temperature distributions.

\begin{tabular}{|c|c|c|c|c|}
\hline $\boldsymbol{T}_{\mathbf{1}}$ & $\boldsymbol{T}_{\mathbf{2}}$ & $\boldsymbol{T}_{\mathbf{3}}$ & $\boldsymbol{T}_{\mathbf{4}}$ & $\boldsymbol{T}_{\mathbf{5}}$ \\
\hline $\mathbf{4 4 0}$ & 660 & 800 & 860 & 840 \\
\hline
\end{tabular}

Table 2: Comparison table between numerical and exact solutions.

\begin{tabular}{|c|c|c|c|c|c|}
\hline \multirow{2}{*}{ 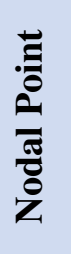 } & \multirow{2}{*}{ 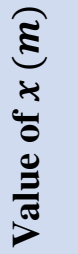 } & \multirow{2}{*}{ 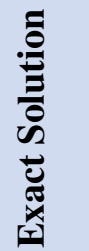 } & \multicolumn{2}{|c|}{ 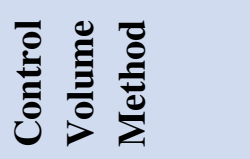 } & \multirow{2}{*}{ 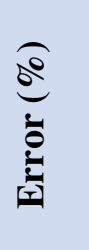 } \\
\hline & & & TDMA & GE & \\
\hline 1 & 0.1 & 440 & 450 & 450 & 2.27 \\
\hline 2 & 0.3 & 660 & 670 & 670 & 1.51 \\
\hline 3 & 0.5 & 800 & 810 & 810 & 1.25 \\
\hline 4 & 0.7 & 860 & 870 & 870 & 1.16 \\
\hline 5 & 0.9 & 840 & 850 & 850 & 1.19 \\
\hline
\end{tabular}

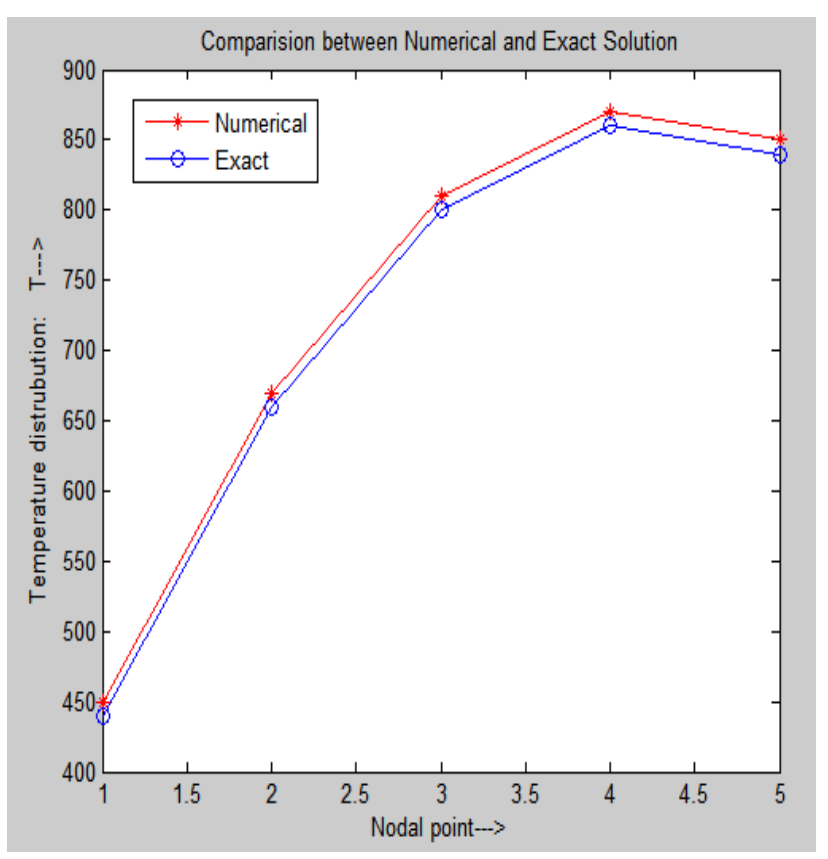

Fig 5: Graphical comparison between control volume method and exact/analytic solution.

\section{CONCLUSION:}

In this work, to investigate one dimensional conductive heat transfer in a uniform plane wall, we have used the finite volume method (FVM). Obtaining discretized equation by FVM, we have applied Tri-Diagonal Matrix Algorithm (TDMA) and Gaussian elimination (GE) method. Finally, with the help of MATLAB R2014a, we represent a tabular and a graphical comparison between our numerical solution and exact solutions. The FVM has given outstanding results which have few errors with respect to exact solution but this error can be minimized by taking more and more grid points. That is, this method is very effective, accurate, reliable and easier to appliance in MATLAB or any other programming languages compared to the other exorbitant methods.

\section{ACKNOWLEDGEMENT:}

We are so much obligated to Md. Sirajul Islam for his patience and thoughtful guidance as a complete supervisor to continue this research work.

\section{CONFLICTS OF INTEREST:}

The authors declare that they have no competing interests with respect to the research.

\section{Nomenclature}

A Control Volume boundary Area $\left(\mathrm{m}^{2}\right)$

$N \quad$ North FVM node

$n \quad$ North Control Volume boundary

$S \quad$ South FVM node

$s \quad$ South Control Volume boundary

$O$ Central Control Volume node

$\psi \quad$ Thermal conductivity $\left(W \mathrm{~m}^{-1} K^{-1}\right)$

$T$ Temperature $\left({ }^{\circ} \mathrm{C}\right)$

a Coefficient of FVM $\left(W K^{-1}\right)$

$q \quad$ Heat generation $\left(\mathrm{kW} \mathrm{m}^{-3}\right)$

$n \quad$ Node

$\delta x \quad$ Distance between nodes $(\mathrm{m})$

$T_{A} \quad$ Refers to temperature at boundary node $\mathrm{A}$

$T_{B} \quad$ Refers to temperature at boundary node B 


\section{REFERENCES:}

1) Berezovski, A. and Maugin, G.A., 2001. Simulation of thermo elastic wave propagation by means of a composite wavepropagation algorithm. Journal of Computational Physics, 168(1), 249-264. https://doi.org/10.1006/jcph.2001.6697

2) Cheniguel, A. and Reghioua, M., 2013, October. On the numerical solution of threedimensional diffusion equation with an integral condition. In Proceedings of the World Congress on Engineering and Computer Science, 2, 23-25.

3) Chuathong, N. and Toutip, W., 2011. An accuracy comparison of solutions between Boundary element method and mesh less method for Laplace equation. In Proceedings of the 16th Annual Meeting in Mathematics (AMM2011), 29, 42.

4) Demirdžić, I. and Muzaferija, S., 1994. Finite volume method for stress analysis in complex domains. International Journal for Numerical Methods in Engineering, 37(21), 3751-3766. https://doi.org/10.1002/nme.1620372110

5) El-Mikkawy, M.E., 2004. A fast algorithm for evaluating nth order tri-diagonal determinants. Journal of computational and applied mathematics, 166(2), 581-584. https://doi.org/10.1016/j.cam.2003.08.044

6) Islam KA, Deeba F, and Hassan MKA. (2019). Dust ion acoustic solitary waves in multi-ion dusty plasma system with adiabatic thermal change, Aust. J. Eng. Innov. Technol., 1(5), 1-5.

https://doi.org/10.34104/ajeit.019.0105

7) Islam MT and Hossain MS. (2019). Hybridization of vigenere technique with the collaboration of RSA for secure communication, Aust. J. Eng. Innov. Technol., 1(6), 6-13.

https://doi.org/10.34104/ajeit.019.06013

8) Jasak, H. and Weller, H.G., 2000. Application of the finite volume method and unstructured meshes to linear elasticity. International journal for numerical methods in engineering, 48(2), 267-287.

9) Lau, M.A. and Kuruganty, S.P., 2010. Spreadsheet implementations for solving boundary-value problems in electromagnetics. Spreadsheets in Education, 4(1), 1-18.

10) Patankar, S.V., 1980. Numerical Heat Transfer and Fluid Flow; Series in Computational and Physical Processes in Mechanics and Thermal Sciences. Taylor \& Francis, 29, 33-34.

11) Patil, P.V. and Prasad, J.K., 2013. Solution of laplace equation using finite element method. International Journal of Science, Spirituality, Business, and Technology, 2(1), 40-46.

12) Sasaki, T. and Murao, H., 1982. Efficient Gaussian elimination method for symbolic determinants and linear systems. ACM Transactions on Mathematical Software (TOMS), 8(3), 277-289. https://doi.org/10.1145/356004.356007

13) Uddin MM, Karim R, Kaysar MA, Dayan MAR, and Islam KA. (2020). Low-cost jutecotton and glass fibre reinforced textile composite sheet, Int. J. Mat. Math. Sci., 2(1), 1-7. https://doi.org/10.34104/ijmms.020.01007

14) Versteeg, H.K. and Malalasekera, W., 2007. An introduction to computational fluid dynamics: the finite volume method. Pearson education.

Citation: Islam S, Islam MS, and Mandal S. (2020). One dimensional heat transfer through a uniform plane wall by using finite volume method, Aust. J. Eng. Innov. Technol., 2(2), 24-30. https://doi.org/10.34104/ajeit.020.024030 (c) 